\title{
Heteroplasmy of chloroplast DNA in Medicago
}

\author{
Lowell B. Johnson ${ }^{1}$ and Jeffrey D. Palmer ${ }^{2}$ \\ ${ }^{1}$ Department of Plant Pathology, Throckmorton Hall, Kansas State University, Manhattan, KS 66506, \\ USA; ${ }^{2}$ Department of Biology, University of Michigan, Ann Arbor, MI 48109-1048, USA
}

Received 15 June 1988; accepted in revised form 27 September 1988

Key words: alfalfa, chloroplast DNA, chloroplast DNA heterogeneity, heteroplasmy, Medicago, restriction mapping

\begin{abstract}
Two chloroplast DNA (cpDNA) regions exhibiting a high frequency of intra- or inter-species variation were identified in 12 accessions of the genus Medicago. Restriction maps of both regions were prepared for alfalfa, and the probable nature of the events causing the DNA differences was identified. Specific DNA fragments were then cloned for use in identification of variants in each region. Two each of M. sativa ssp. varia and ssp. caerulea and one of six $M$. sativa ssp. sativa single plants examined possessed cpDNA heterogeneity as identified by screening extracts for fragments generated by the presence and absence of a specific $X b a \mathrm{I}$ restriction site. Three plants of $M$. sativa ssp. sativa, two of each of sspp. varia and caerulea, and three $M$. scutellata were also examined for single-plant cpDNA heterogeneity at a hypervariable region where differences resulted from small insertion-deletion events. A single $M$. scutellata plant with mixed cpDNAs was identified. Sorting out was seen when one ssp. sativa plant with mixed plastid types identifiable by the $X b a$ I restriction site difference was vegetatively propagated. This indicated that the initial stock plant was heteroplastidic. Controlled crosses will be required in order to test whether heteroplasmy results from chloroplast transmission in the pollen and to examine the dynamics of sorting out. However, heteroplasmy is apparently not a rare situation in Medicago.
\end{abstract}

\section{Introduction}

Cells of flowering plants possess many copies of their chloroplast genome $[1,3,4]$, in contrast to only one or a few copies, depending on ploidy level, of their nuclear genome. Chloroplast genome numbers are estimated to range from 1900 to 50000 copies per cell, with multiplicity occurring in both plastid number and DNA copies per plastid [1]. It is generally observed, however, that single plants exhibit homogeneity of chloroplast DNA (cpDNA),

Contribution No 88-547-J from the Kansas Agricultural Experiment Station, Manhattan. presumably as the result of a relatively rapid "sorting out" of plastids by vegetative segregation $[3,4]$. Examples of this plastid sorting out have been clearly established in studies with variegated plants $[4,9,30]$ and with somatic hybrid plants regenerated following protoplast fusion $[8,23]$.

There are a few exceptions, however, to the above generalizations. For example, Moon et al. [16] claimed the existence of a persistent cpDNA heterogeneity in single rice plants. Lax et al. [10] have described a plastome mutant of cotton that exhibits a slow rate of sorting out, resulting in a persistence of mixed cells possessing both wild type and mutant plastids. Heteroplasmy is possible in several species 
of conifers that exhibit paternal plastid inheritance $[33,34]$. In contrast to the apparent rarity of heteroplasmy in cpDNA, numerous cases of heteroplasmic mitochondrial DNAs have been reported in animals (reviewed in [31]).

We became interested in the possibility of locating heteroplastidic plants in alfalfa, Medicago sativa L., after a report from our laboratory by Rose et al. [24] documenting the recovery of one or the other of two types of cpDNA in 23 plants regenerated from protoplasts (protoclones). Results were most easily explained if the original, seed-derived protoplast donor plants possessed both types of cpDNA, but with sorting out having occurred in individual shoots, probably prior to protoplast isolation.

Medicago would seem to be a logical plant genus within which to look for at least occasional singleplant cpDNA heterogeneity. Studies by Smith et al. [30], using two chlorophyll-deficiency mutants in alfalfa, provided developmental and genetic evidence suggesting that both were plastid-encoded mutations and that there was a high frequency of transmission of these mutant plastids through the pollen. There is also cytological evidence for biparental inheritance of plastids in alfalfa [6]. An earlier report of biparental plastid transmission exists for M. truncatula [12]. The biparental transmission of cpDNA in Medicago should provide a mechanism for reestablishing cpDNA heterogeneity.

CpDNAs of the genus Medicago are also characterized by the lack of the large inverted repeat ([20], Johnson and Palmer, unpublished) that is found in most other land plant cpDNAs. The absence of this repeat correlates with an increased frequency of plastid DNA evolutionary rearrangement [22], and nucleotide substitution is significantly reduced in the inverted repeat of cpDNAs relative to single copy regions [36]. Thus studies involving a genus lacking this repeat structure might provide a greater number of mutants with cpDNAs readily distinguishable by restriction patterns with which to work.

In this paper, the identification and characterization of several cpDNA regions with a high frequency of intraspecific and interspecific variability in the genus Medicago is described. Cloned fragments from these regions are then used to demonstrate singleplant cpDNA heterogeneity and heteroplasmy.

\section{Materials and methods}

Medicago accessions obtained from the USDA Regional Plant Introduction Station, Ames, Iowa, were $M$. lupulina L. (PI289310), M. orbicularis (L.) Bart. (PI385011), M. sativa L. ssp. sativa (PI26590), $M$. sativa L. ssp. falcata (PI234815), $M$. sativa L. ssp. varia (PI238145), $M$. sativa L. ssp. caerulea (PI206453), and $M$. cancellata Marschall von Bieberstein (PI315458). M. carstiensis Wulf. (TM993), M. arborea L. (UAG1497), and M. papillosa Boiss. ssp. papillosa (UAG98), initially designated $M$. dzhawakhetica [29], were provided by T. McCoy, University of Arizona, Tucson. E. L. Sorensen, Kansas State University, provided seed for $M$. sativa $L$. ssp. falcata cv. Anik, as did I. D. Kaehne, Dept. of Agriculture and Fisheries, Adelaide, South Australia, for $M$. scutellata cv. Robinson. Several Regen S alfalfa [2] clones and protoclones, including RS-K1, RS-K2, and K2-66S, were utilized, and have been maintained by propagation of shoot cuttings since the initial studies of their cpDNA by Rose et al. [24].

Chloroplast DNAs from bulked plant samples were extracted from plastids banded in sucrose gradients and then pelleted by centrifugation $[18,20]$. Following plastid lysis, cpDNAs were banded twice in $\mathrm{CsCl}$ gradients, dialyzed, and stored at $4{ }^{\circ} \mathrm{C}[18$, 20]. The sucrose gradient step was typically omitted from the procedure for single-plant extracts.

Restriction endonuclease digestion, 0.7 or $1.0 \%$ agarose slab gel electrophoresis, ethidium bromide staining and gel photography, blotting to Zetabind (AMF CUNO, Meriden, CT), nick translation, filter hybridization, and autoradiography were done after Palmer $[18,20]$. Probes used included a cloned set of eight alfalfa [20] and four mung bean [20, 21] fragments spanning the entire chloroplast genome.

Several smaller subclones were prepared from the aforementioned alfalfa probes by digestion, electrophoresis, and excision of the desired subfragment from an agarose gel, followed by electroelution, phenol and diethyl ether extraction, and ethanol precipitation [18]. Fragments were next ligated into the appropriately cut $\mathrm{pIC} 20 \mathrm{H}$ vector [15], which was then transformed into competent cells of Escherichia coli strain JM83. Recombinant white colonies were then selected on ampicillin/X gal plates, 
and inserts characterized from plasmid DNA minipreps [5].

\section{Results}

The first cpDNA polymorphism selected for study involved the $X b a$ I region suggested by Rose et $a l$. [24] to reveal heteroplasmy in certain Regen S alfalfa donor clones. A preliminary comparison of $X b a \mathrm{I}$ fragmentation patterns of the cpDNAs of bulk extracts of single accessions of $M$. sativa ssp. sativa, ssp. varia, and ssp. caerulea revealed a reduced ethidium bromide staining in gels of cpDNAs of these three subspecies for the same $7.8 \mathrm{~kb}$ fragment observed variable by Rose et al. [24] relative to other singlet bands such as the one at $7.5 \mathrm{~kb}$ (Fig. 1A). Increased intensity of staining also occurred within a triplet of bands of 8.3 to $8.5 \mathrm{~kb}$. This suggested heter-

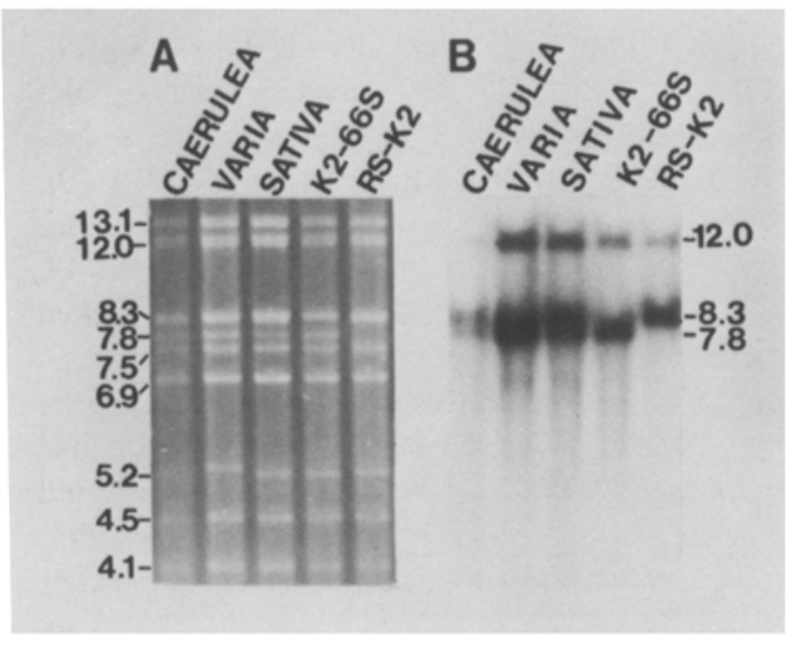

Fig. 1. Evidence of cpDNA polymorphism in Medicago sativa populations following $X b a$ I digestion. From left to right, ssp. caerulea, ssp. varia, ssp. sativa PI26590, ssp. sativa K2-66S, and ssp. sativa RS-K2. (A) Ethidium bromide-stained gels showing possible heterogeneity as a consequence of variable staining of a $7.8 \mathrm{~kb}$ fragment relative to other singlet bands such as the one at $7.5 \mathrm{~kb}$ for the first three accessions. Increased staining intensity in a triplet of bands at 8.3 to $8.5 \mathrm{~kb}$ suggested involvement of one of these fragments in this heterogeneity. (B) Autoradiogram of blot from this same gel following hybridization with the alfalfa $6.2 \mathrm{~kb}$ cpDNA fragment of Palmer et al. [20]. Fragments of both 8.3 and $7.8 \mathrm{~kb}$ from sspp. caerulea, varia, and sativa PI26590 hydridized to this probe, thus revealing cpDNA heterogeneity within each population. ogeneity in these subspecies, prompting further investigation and ultimately single plant analyses.

The variable fragment was first located on the alfalfa cpDNA restriction map of Palmer et al. [20] by hybridization with cloned fragments that cover the alfalfa chloroplast genome. These include eight alfalfa $P$ st $\mathrm{I}$ fragments and four mung bean fragments that span the single uncloned alfalfa $P$ st I fragment of $34 \mathrm{~kb}$. These clones were hybridized with blots containing $X b a$ I digests of cpDNAs of protoclone $\mathrm{K} 2-66 \mathrm{~S}$ possessing the fragment [24] and a recently located RS-K2 plant lacking the fragment (Fig. 1A, B). The $6.2 \mathrm{~kb}$ alfalfa probe clearly showed the location of the difference, hybridizing to an $8.3 \mathrm{~kb} \mathrm{Xba}$ I fragment with the RS-K2 extract and the 0.5 (not shown) and $7.8 \mathrm{~kb}$ fragments with the K2-66S extract. Without the $X b a$ I site under study, the slightly larger $8.3 \mathrm{~kb}$ fragment comigrated with several other fragments of 8.3 to $8.5 \mathrm{~kb}$. Further mapping studies indicated that the $X b a \mathrm{I}$ difference under study was due to a single restriction site change, presumably the result of a single base pair substitution, because no differences were observed with any of the other nine enzymes whose sites were mapped (Fig. 2A). However, the possibility of a small insertion, deletion or inversion altering or removing the site involved, yet causing an imperceptible change in the size of the $0.8 \mathrm{~kb} E c o \mathrm{RI}$ fragment, the smallest fragment studied containing the site, cannot be eliminated.

In order to simplify the interpretation of autoradiograms from single plant extracts, a smaller probe was prepared by subcloning a $3.2 \mathrm{~kb} \mathrm{Xba} \mathrm{I-Sac}$ I fragment from the cloned $\boldsymbol{P}_{\text {St }}$ I $6.2 \mathrm{~kb}$ fragment of Palmer et al. [20]. As predicted from the map shown in Fig. 2A, this subclone hybridized to a $3.2 \mathrm{~kb} X b a$ I-Sac I fragment when the $X b a$ I site was missing and to $2.7 \mathrm{~kb}$ and $0.5 \mathrm{~kb}$ fragments when the site was present. It likewise hybridized to the expected 8.3 , and 7.8 and $0.5 \mathrm{~kb}$ fragments, respectively, when $\mathrm{Sac}$ I was omitted from the digestions. When blots of bulk extracts of cpDNAs of sspp. sativa, varia and caerulea were hybridized with either the $6.2 \mathrm{~kb}$ (Fig. 1B) or the $3.2 \mathrm{~kb}$ probes, all three showed evidence of heterogeneity. In the case of varia, most hybridization was to the $7.8 \mathrm{~kb}$ fragment, but it was clear when single plants were examined that heter- 


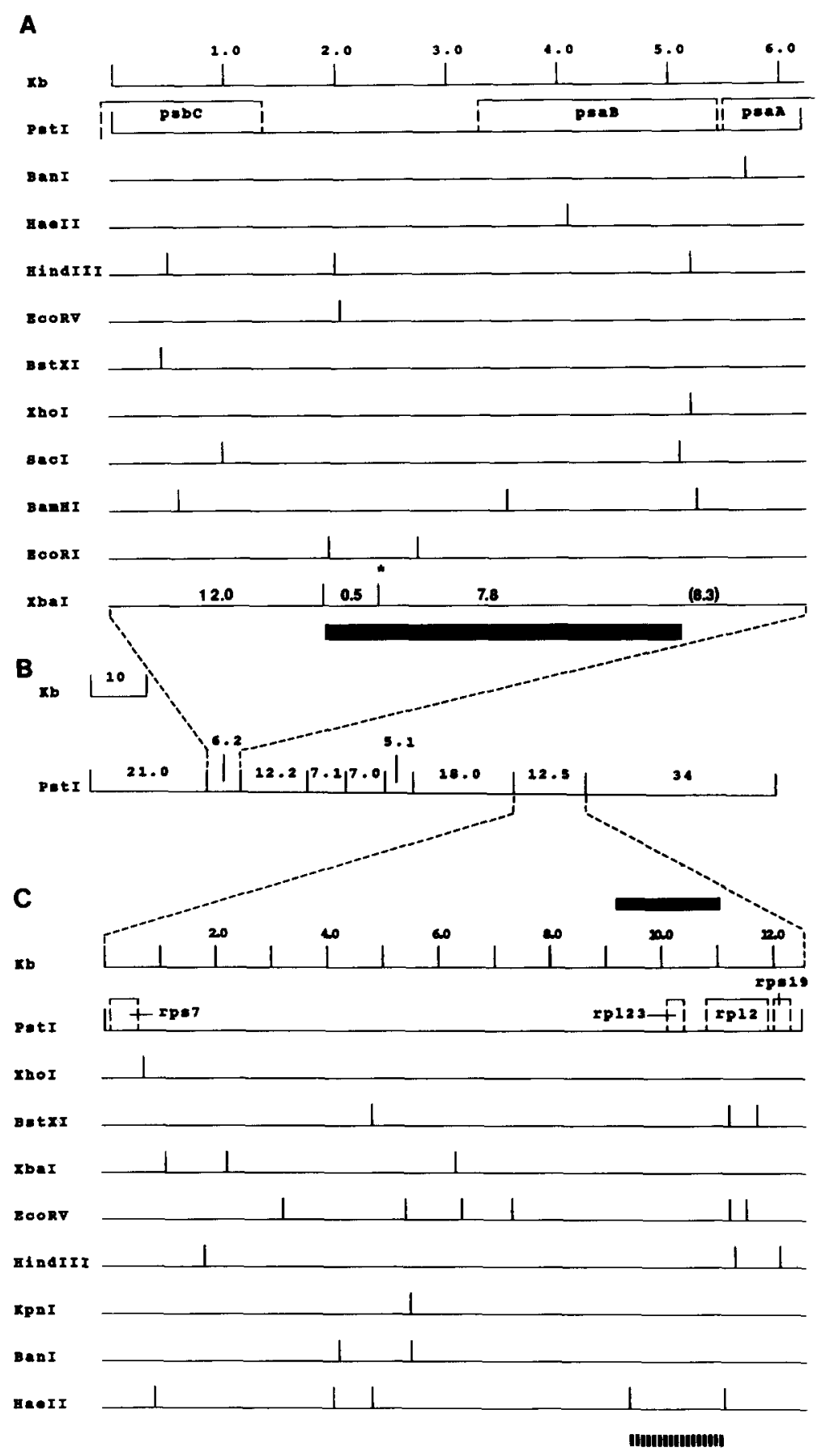

Fig. 2. (A) Restriction map of the $6.2 \mathrm{~kb}$ Pst I fragment of alfalfa cpDNA for 10 additional enzymes. The fragment contains no $B s t$ EII or $K p n$ I sites. Shown in bold numbers are the sizes in kb of the $X b a$ I fragments. The $X b a$ I site marked by an asterisk (*) is responsible for the polymorphism under study. When present, 0.5 and $7.8 \mathrm{~kb}$ fragments occur. When absent, an $8.3 \mathrm{~kb}$ fragment (in parentheses) is seen instead. The solid bar indicates the extent of the $3.2 \mathrm{~kb} \mathrm{Xba} \mathrm{I-Sac} \mathrm{I} \mathrm{fragment} \mathrm{used} \mathrm{as} \mathrm{a} \mathrm{hybridization} \mathrm{probe} \mathrm{in} \mathrm{Fig.} \mathrm{3.} \mathrm{Approximate}$ gene positions are from the unpublished mapping data of J. Palmer. (B) Linearized map of alfalfa cpDNA Pst I fragments after Palmer et al. [20]. (C) Restriction map of the $12.5 \mathrm{~kb}$ Pst I fragment of alfalfa cpDNA for eight additional enzymes. The fragment contains no Bst EII or Sac I sites. The vertically striped bar below the Hae II map delineates the hypervariable region of interest. The solid bar above the map indicates the approximate location of the $1.9 \mathrm{~kb} \mathrm{Bam} \mathrm{HI} \mathrm{fragment} \mathrm{used} \mathrm{as} \mathrm{a} \mathrm{hybridization} \mathrm{probe} \mathrm{in} \mathrm{Fig.} \mathrm{5.} \mathrm{Approximate} \mathrm{gene}$ positions are from the unpublished mapping data of J. Palmer. 


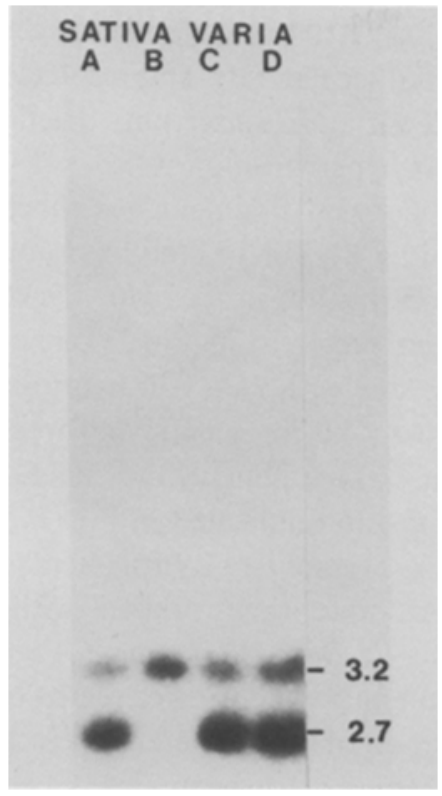

Fig. 3. Evidence of single plant heterogeneity of cpDNA in Medicago, as shown in an autoradiogram of a gel blot containing cpDNA double-digested with $X b a \mathrm{I}$ and Sac I. Hybridization was with a nick-translated, cloned $3.2 \mathrm{~kb} X b a \mathrm{I}-\mathrm{Sac}$ I fragment (Fig. 2) derived from the alfalfa $6.2 \mathrm{~kb}$ Pst I fragment of Palmer et al. [20]. Estimated fragment sizes in $\mathrm{kb}$ are shown on the right. The region of the autoradiogram shown is from the origin to about $2.4 \mathrm{~kb}$. Lanes A and B contained digests from single $M$. sativa ssp. sativa plants, lanes $C$ and $D$ digests from single $M$. sativa ssp. varia plants.

ogeneity existed within the accession (Fig. 3).

A small number of single plant DNAs were then double digested with $X b a$ I and $S a c$ I and the resulting fragments separated by electrophoresis. Filter blots containing the restriction fragments were then hybridized with the $3.2 \mathrm{~kb}$ probe. One of two ssp. sativa plants and each of two ssp. varia plants provided clear evidence of heterogeneity at this $X b a$ I site (Fig. 3), as did each of two plants of ssp. caerulea (data not shown). Note that digestion was complete, as evidenced by both the total lack of hybridization of the $3.2 \mathrm{~kb}$ probe to any fragment larger than 3.2 or $2.7 \mathrm{~kb}$ (Fig. 2) and by the hybridization of other clones to only those $X b a$ I fragments expected in a complete digest. In addition, this heterogeneity was reproducible in several different digestions of the same DNA, digests that were otherwise complete by the criteria just mentioned. Therefore we feel that this heterogeneity is not an artifact of partial digestion, but is real, resulting from single plants containing two populations of cpDNA molecules, one with and one without the $X b a$ I site marked by an asterisk in Fig. 2A. A later extraction of four different ssp. sativa plants revealed one possessing a $3.2 \mathrm{~kb}$ fragment and three with a $2.7 \mathrm{~kb}$ fragment, but no heterogeneity.

Plant A of ssp. sativa was propagated by rooting of two shoot tip cuttings, and the resultant plants were tested for cpDNA heterogeneity. Hybridization experiments revealed that both plants contained detectable amounts of only the $2.7 \mathrm{~kb}$ fragment, the predominant one in the original plant $\mathrm{A}$, documenting the eventual occurrence of sorting out $[3,4,9$, 30].

The second variable region of alfalfa cpDNA that we selected for study was initially identified during efforts to determine phylogenetic relationships within the genus Medicago through the use of cpDNA

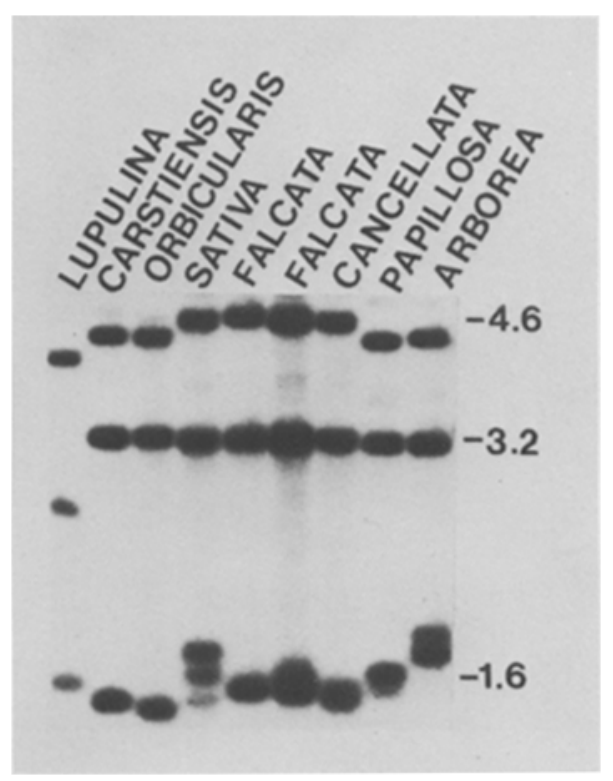

Fig. 4. Hypervariable (HV) region in cpDNA from nine Medicago accessions, as seen in a portion of an autoradiogram of a gel blot containing Hae II-digested cpDNA. Hybridization was with a cloned, nick-translated $12.5 \mathrm{~kb} P_{s t}$ I fragment from alfalfa cpDNA [20]. Estimated fragment sizes in $\mathrm{kb}$ are shown on the right. Accessions from left to right are M. lupulina, $M$. carstiensis, $M$. orbicularis, $M$. sativa ssp. sativa, $M$. sativa ssp. falcata, $M$. sativa ssp. falcata cv. Anik, $M$. cancellata, $M$. papillosa, and $M$. arborea. The HV region contains fragments of approximately 1.45 to $1.8 \mathrm{~kb}$ among the accessions shown. 
restriction mapping (L. B. Johnson and J. D. Palmer, unpublished). Figure 4 shows the hybridization of a $12.5 \mathrm{~kb}$ alfalfa clone (Fig. 2B) to Hae II digests of cpDNAs from a group of Medicago accessions. Two regions of fragment size polymorphism are obvious. A region of interspecific variation only is located within a group of fragments of 4.0 to $4.6 \mathrm{~kb}$ and was not studied further. The second region, encompassing a nearly continuous range of size forms from 1.45 to $1.8 \mathrm{~kb}$, exhibited both interspecific and intraspecific variation (Fig. 4). The cpDNAs in this figure were prepared from approximately 30 to 100 pooled plants, and it was clear that not all fragments were equally represented within those mixed populations, e.g. ssp. sativa. A similar pattern of fragment size variation as revealed by Hae II digestion (Fig. 4) was also documented with several other enzymes (Ava I, Bam HI, Cla I, Eco RV) that produce relative-

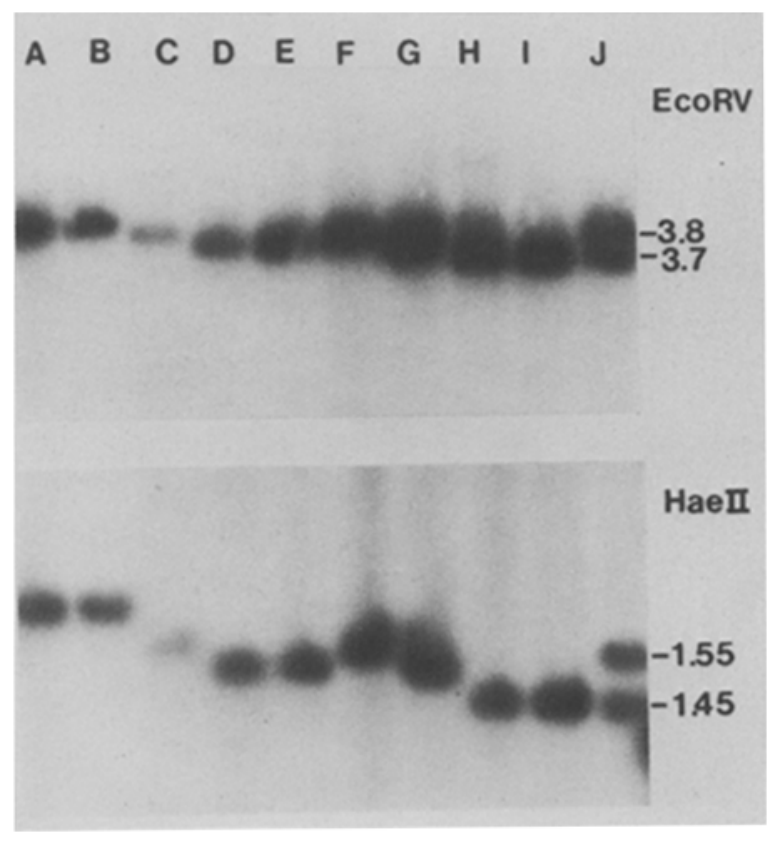

Fig. 5. Evidence of single plant heterogeneity of cpDNA in Medicago, as shown in autoradiograms of gel blots containing single-plant cpDNAs digested with Eco RV (top) or Hae II (bottom). Hybridization was with a nick-translated, cloned $1.9 \mathrm{~kb}$ Bam $\mathrm{HI}$ fragment derived from the alfalfa $12.5 \mathrm{~kb} P_{S t}$ I fragment of Palmer et al. [20]. Estimated fragment sizes in $\mathrm{kb}$ are shown on the right. Single plant digests for both enzymes include $M$. sativa ssp. sativa (lanes A-C), $M$. sativa ssp. varia (lanes D and E), $M$. sativa ssp. caerulea (lanes $\mathrm{F}$ and $\mathrm{G}$ ) and $M$. scutellata (lanes $\mathbf{H}-\mathbf{J}$ ). Single plant heterogeneity is seen in lane $J$. ly small fragments in this region of the genome (data not shown; but see Fig. 5B). This result clearly implicates length variation (insertions/deletions) as the cause of this hypervariation.

A $1.9 \mathrm{~kb} \mathrm{Bam}$ HI fragment was subcloned from the parent $12.5 \mathrm{~kb}$ Pst I clone to produce a more specific hybridization probe. The $1.9 \mathrm{~kb}$ fragment has not been precisely mapped, but with alfalfa cpDNA digested with Hae II it hybridizes strongly to the 1.5 to $1.7 \mathrm{~kb}$ fragments, and weakly only to the adjacent $4.6 \mathrm{~kb}$ fragment. This places it in the approximate position indicated in Fig. 2C.

Blots of Hae II and Eco RV fragments from single plant DNAs were then probed with the nicktranslated $1.9 \mathrm{~kb}$ Bam $\mathrm{HI}$ fragment. Figure 5 shows hybridization patterns for three plants of $M$. sativa ssp. sativa (two of which were used in Fig. 3), two sativa ssp. varia (both used in Fig. 3), two sativa ssp. caerulea, and three $M$. scutellata. None of the sativa subspecies shown exhibited single-plant heterogeneity for these fragments under study. However, cpDNA of one $M$. scutellata (plant $J$ ) is clearly heterogeneous in this region, possessing roughly equal amounts of the two Hae II fragments (1.45 and 1.55 $\mathrm{kb})$ and two $E c o$ RV fragments ( 3.7 and $3.8 \mathrm{~kb}$ ). This heterogeneity was demonstrable with both the 1.9 and $12.5 \mathrm{~kb}$ probes. The cpDNA possessing the larger of these two fragments must have been relatively rare in our $M$. scutellata population, in that the fragment was not observed in blots from bulk extracted cpDNA (data not shown).

\section{Discussion}

Our results clearly establish that single plants in the genus Medicago can possess more than one type of plastid DNA. This heterogeneity was of two types. In the first type, the two DNAs were distinguishable using $X b a \mathrm{I}$, and differed by a single restriction site, possibly resulting from a single base pair change. Heteroplasmic plants possessing both the $X b a$ I forms (Figs. 1, 3) were identifiable in three different subspecies of sativa, and plants with only one form or the other were also found. Sorting out was demonstrated, as was apparently also observed by Rose et al. [24]. Thus heteroplasmy is clearly in- 
volved. Its high frequency of occurrence strongly suggests its perpetuation by pollen transmission, although if some mechanism existed to maintain heteroplasmy at levels below our detection limit, these two $X b a$ I variants might conceivably crop up repeatedly in different plants.

The second type of cpDNA heterogeneity observed was the result of frequent insertion or deletion events that have occurred both within and between species (Fig. 4). Nine individual plants of three subspecies of $M$. sativa were all monomorphic at the size-variable locus, even though size heterogeneity was present in the single population studied. We detected single-plant heteroplasmy at this locus in only one $M$. scutellata plant (Fig. 5). Reasons for the low frequency of single-plant heteroplasmy in the sativa subspecies relative to that of the $X b a I$ variants are unclear, although it may be a reflection of chance, given the relatively small sample number.

There is little likelihood that either nuclear or mitochondrial DNAs contribute to the polymorphisms under question, even though the presence of cpDNA in both nuclear $[26,35]$ and mitochondrial $[7,13,14,16,26,28,32,35]$ genomes of plants is well documented. Nuclear DNA can be ruled out in the $M$. sativa ssp. sativa plant A that initially possessed both forms of $X b a$ I-variant molecules, since somatic sorting out occurred as a result of vegetative propagation. In addition, several of our mixed plants (e.g. M. scutellata plant J) contained nearly equimolar amounts of the two DNAs. This is a most unlikely occurrence if one fragment came from either the nuclear or mitochondrial genomes, considering the high copy number of cpDNA molecules in leaves [1] and the several-fold enrichment of cpDNA in our extraction procedure. It is also clear, considering the hypervariable, length-polymorphic region, that most plants contained only one of the many different fragments, which always clearly mapped to the chloroplast genome.

The nature of the DNA sequences responsible for the frequent size variation in the hypervariable region of our Medicago accessions is unknown. One possible explanation for this variation is that the region contains a short tandem repeat element that undergoes copy number changes via unequal crossover to produce the observed diversity. There is precedent for this phenomenon with other cpDNAs. A tandem repeat was sequenced in Euglena that produces a high degree of size polymorphism within that region [25]. Pea also possesses a size variable region hypothesized by Palmer et al. [19] to result from a short tandem repeat. In addition, as cited by Solignac et al. [31], numerous studies have documented that heteroplasmy in animal mtDNA results from variable number tandem repeats. Sequencing of this hypervariable region in cpDNAs from several of our $M$. sativa ssp. sativa plants, where the variation occurs within a single populations of plants, could do much to reveal mechanisms involved.

Our results were obtained with a random sample of plants, most selected from populations containing cpDNA heterogeneity. From our small population it is difficult to assess how frequently singleplant cpDNA heteroplasmy exists in Medicago. We assume that the phenomenon stems in large part from the reported biparental transmission of plastids $[6,30]$, which occurs at high frequency in alfalfa [30], and should at least initially manifest as heteroplasmy at the single-cell level. Controlled crosses between plants of differing cpDNA types will be required before the frequency of this phenomenon can ultimately be assessed.

It is noteworthy that alfalfa is a perennial and largely self-sterile outcrosser, while $M$. scutellata is a self-pollinated annual [11], yet both exhibited single-plant cpDNA heterogeneity in our study. $M$. truncatula, another annual [11], like alfalfa [6, 30] has also been reported to exhibit biparental plastid inheritance [12]. Based on these observations it is interesting to speculate that either heteroplasmy or the preservation of plastid variation may confer some evolutionary advantage in Medicago. Alternatively, pollen plastid transmission is of trivial importance in alfalfa and presumably other perennial Medicago species, and was merely retained during the postulated evolutionary divergence of the annuals from the perennials. Should M. scutellata not possess plastid biparental inheritance, the heteroplastidic plant observed may simply possess a recent mutation that ultimately would sort out, and as such may be a reflection of the extreme hypervariability of this particular region of the genome.

The alfalfa cpDNA heterogeneity that we ob- 
served appears different from that in rice in that we have observed sorting out. In rice, single plants were reported by Moon et al. [16] to maintain a relatively constant rate of two cpDNA types, although evidence for both forms of the putative rice cpDNA being of true chloroplast origin is not entirely convincing [17]. Lax et al. [10] studied sorting out in variegated cotton containing a plastome mutant, and suggested that the high frequency of plants heterozygous for cpDNA was the result of a slow sorting out, possibly facilitated by plastid fusions that produced within-plastid DNA heterogeneity. We could not measure sorting out rate, both because our cpDNA mutants did not result in chlorophylldeficient plants and because it is unlikely that all plants assayed were even fertilized by pollen possessing a different cpDNA type.

\section{Acknowledgements}

This research was supported by a grant to J. D. Palmer from the National Institutes of Health (GM35087) and by the Kansas Agricultural Experiment Station, Manhattan, KS. The assistance of E. Small in confirming the identity of most accessions used in this study is gratefully acknowledged.

\section{References}

1. Bendich $\mathrm{AJ}$ : Why do chloroplasts and mitochondria contain so many copies of their genome? BioEssays 6: 279-282 (1987).

2. Bingham ET, Hurley LV, Kaatz DM, Saunders JW: Breeding alfalfa which regenerates from callus tissue in culture. Crop Sci 15: 719-721 (1975).

3. Birky CW Jr: Transmission genetics of mitochondria and chloroplasts. Annu Rev Genet 12: 471-512 (1978).

4. Birky CW Jr: Relaxed cellular controls and organelle heredity. Science 222: $468-475$ (1983).

5. Birnboim HC, Doly J: A rapid alkaline extraction procedure for screening recombinant plasmid DNA. Nucl Acids Res 7: $1513-1523$ (1979).

6. Corriveau JL, Coleman AW: Rapid screening method to detect potential biparental inheritance of plastid DNA and results for over 200 angiosperm species. Am J Bot 75: 1443-1458 (1988).

7. Dron M, Hartmann C, Rode A, Sevignac M: Gene conversion as a mechanism for divergence of a chloroplast tRNA gene inserted in the mitochondrial genome of Brassica oleracea.
Nucl Acids Res 13: 8603 - 8610 (1985).

8. Fluhr R: The segregation of organelles and cytoplasmic traits in higher plant somatic fusion hybrids. In: Potrykus I, Harms CT, Hinnen A, Hutter R, King PJ, Shillito RD (eds) Protoplasts 1983 Lecture Proc, pp. 85-92. Birkhauser Press, Basel (1983).

9. Kirk JTO, Tilney-Bassett RAE: The Plastids: Their Chemistry, Structure, Growth and Inheritance, rev 2nd edn. Elsevier/North-Holland Biomedical Press, Amsterdam/New York/Oxford (1978).

10. Lax AR, Vaughn KC, Duke SO, Endrizzi JE: Structural and physiological studies of a plastome cotton mutant with slow sorting out. J Hered 78: 147-152 (1987).

11. Lesins KA, Lesins I: Genus Medicago (Leguminosae): A Taxogenetic Study. Dr. W Junk, The Hague (1979).

12. Lilienfeld FA: Plastid behavior in reciprocally different crosses between two races of Medicago truncatula Gaertn. Seiken Ziho 13: 3-38 (1962).

13. Lonsdale DM, Hodge TP, Howe CJ, Stern DB: Maize mitochondrial DNA contains a sequence homologous to the ribulose-1,5-bisphosphate carboxylase large subunit gene of chloroplast DNA. Cell 34: 1007-1014 (1983).

14. Marechal L, Runeberg-Roos P, Grienenberger JM, Colin J, Weil JH, Lejeune B, Quetier F, Lonsdale DM: Homology in the region containing a tRNA ${ }^{\mathrm{Trp}}$ gene and a (complete or partial) $t R_{N A}{ }^{P r o}$ gene in wheat mitochondrial and chloroplast genomes. Curr Genet 12: 91-98 (1987).

15. Marsh JL, Erfle M, Wykes EJ: The pIC plasmid and phage vectors with versatile cloning sites for recombinant selection by insertional inactivation. Gene 32: 481-485 (1984).

16. Moon E, Kao T-H, Wu R: Rice chloroplast DNA molecules are heterogeneous as revealed by DNA sequences of a cluster of genes. Nucl Acids Res 15: 611-630 (1987).

17. Nishizawa J, Hirai A: Nucleotide sequence and expression of the gene for the large subunit of rice ribulose-1,5bisphosphate carboxylase. Japan J Genet 62: 389-395 (1987).

18. Palmer JD: Isolation and structural analysis of chloroplast DNA. Methods Enzymol 118: 167-186 (1986).

19. Palmer JD, Jorgensen RA, Thompson WF: Chloroplast DNA variation and evolution in Pisum: Patterns of change and phylogenetic analysis. Genetics 109: 195-213 (1985).

20. Palmer JD, Osorio B, Aldrich J, Thompson WF: Chloroplast DNA evolution among legumes: Loss of a large inverted repeat occurred prior to other sequence rearrangements. Curr Genet 11: 275-286 (1987).

21. Palmer JD, Thompson WF: Clone banks of the mung bean, pea and spinach chloroplast genomes. Gene 15:21-26(1981).

22. Palmer JD, Thompson WF: Chloroplast DNA rearrangements are more frequent when a large inverted repeat sequence is lost. Cell 29: $537-550$ (1982).

23. Pelletier GR: Plant organelle genetics through somatic hybridization. Oxford Surveys Plant Mol Cell Biol 3: 97-121 (1986).

24. Rose RJ, Johnson LB, Kemble RJ: Restriction endonuclease studies on the chloroplast and mitochondrial DNAs of alfal- 
fa (Medicago sativa L.) protoclones. Plant Mol Biol 6: 331-338 (1986).

25. Schlunegger B, Stutz E: The Euglena gracilis chloroplast genome: structural features of a DNA region possibly carrying the single origin of DNA replication. Curr Genet 8: 629-634 (1984).

26. Schuster W, Brennicke A: Plastid, nuclear and reverse transcriptase sequences in the mitochondrial genome of Oenothera: is genetic information transferred between organelles via RNA? EMBO J 6: 2857-2863 (1987).

27. Scott NS, Timmis JN: Homologies between nuclear and plastid DNA in spinach. Theor Appl Genet 67: 279-288 (1984).

28. Sederoff RR, Ronald P, Bedinger P, Rivin C, Walbot V, Bland $M$, Levings III CS: Maize mitochondrial plasmid Ssequences share homology with chloroplast gene PSBA. Genetics 113: 469-482 (1986).

29. Small E: A clarification of the Medicago papillosa complex. Can J Bot 64: 2800-2806 (1986).

30. Smith SE, Bingham ET, Fulton RW: Transmission of chlorophyll deficiencies in Medicago sativa. Evidence for biparental inheritance of plastids. J Hered 77: $35-38$ (1986).

31. Solignac M, Genermont J, Monnerot M, Mounolou J-C: Drosophila mitochondrial genetics: Evolution of heteroplasmy through germ line cell divisions. Genetics 117: 687-696 (1987).

32. Stern DB, Palmer JD: Extensive and widespread homologies between mitochondrial and chloroplast DNA in plants. Proc Natl Acad Sci USA 81: 1946-1950 (1984).

33. Szmidt AE, Alden T, Hallgren J-E: Paternal inheritance of chloroplast DNA in Larix. Plant Mol Biol 9: 59-64 (1987).

34. Wagner DB, Furnier GR, Saghai-Maroof MA, Williams SM, Dancik BP, Allard RW: Chloroplast DNA polymorphisms in lodgepole and jack pines and their hybrids. Proc Natl Acad Sci USA 84: 2097-2100 (1987).

35. Whisson DL, Scott NS: Nuclear and mitochondrial DNA have sequence homology with a chloroplast gene. Plant Mol Biol 4: 267-273 (1985).

36. Wolfe KH, Li W-H, Sharp PM: Rates of nucleotide substitution vary greatly among plant mitochondrial, chloroplast, and nuclear DNAs. Proc Natl Acad Sci USA 84: 9054-9058 (1987). 